\title{
Introduction to Special Issue: State of the Art Reviews on Mental Health in Traumatic Brain Injury
}

\author{
Grahame K. Simpson ${ }^{1,2}$ and Robyn Tate ${ }^{2}$ \\ ${ }^{1}$ Brain Injury Rehabilitation Research Group, Ingham Institute for Applied Medical Research and Liverpool \\ Brain Injury Rehabilitation Unit, Liverpool Hospital, Sydney, Australia \\ ${ }^{2}$ Rehabilitation Studies Unit, Sydney School of Medicine, University of Sydney, Australia
}

Addressing mental health issues is a core concern in the treatment of people with traumatic brain injury (TBI) and their families. Mental health issues are widespread after TBI and arise whether the injury was sustained in civilian or conflict contexts. In the Guest Editorial, Simon Fleminger outlines key issues and conundrums that arise in the intertwining of mental health problems and TBI, as well as the importance of integrating the treatment of mental health conditions into neurorehabilitation.

The Special Issue seeks to advance this process of closer integration by presenting a collection of state of the art reviews on mental health in TBI. The contributions highlight the ongoing evolution of the science of evidence-based reviews. Developments such as the publication of the PRISMA statement have helped set ever higher benchmarks for transparency and rigour in the reporting of reviews. Furthermore, reviews have proven to be versatile, able to evaluate a range of elements that contribute to best practice in mental health care. For example, contributions in this Special Issue canvass the current evidence base of treatment interventions for co-morbid mental health conditions after TBI, the strength of evidence for various prognostic factors in predicting psychological distress, the sensitivity of signs, symptoms or assessment tools in the diagnosis of co-morbid mental health disorders and the prevalence rates for specific mental health conditions.

Results from the current collection highlight the inestimable value of well-conducted reviews. Authors face a series of daunting tasks: to sort through a complex and heterogeneous set of articles; to identify and collate the body of knowledge addressing a specific clinical question; to synthesise the findings; and to distil the essence of the results from the most highly rated articles. Out of this process come findings that educate, bringing greater clarity to areas of clinical practice in which ignorance, confusion or uncertainty have reigned. The findings can also challenge, highlighting important areas for improvement of research methodology, as well as identifying gaps in the knowledge base that provide direction for future research. We hope that the articles in this Special Issue reach all these goalposts in addressing the complex field of mental health and TBI. We commend them to you all. 